\title{
CD90/THY1 is overexpressed in prostate cancer-associated fibroblasts and could serve as a cancer biomarker
}

Lawrence D True ${ }^{1}$, Hui Zhang, ${ }^{2,9}$, Mingliang $\mathrm{Ye}^{2,10}$, Chung-Ying Huang ${ }^{3}$, Peter S Nelson ${ }^{4}$, Priska D von Haller ${ }^{5,11}$, Larry W Tjoelker ${ }^{5,12}$, Jong-Seo Kim ${ }^{6}$, Wei-Jun Qian ${ }^{6}$, Richard D Smith ${ }^{6}$, William J Ellis ${ }^{7}$, Emily S Liebeskind ${ }^{7,8}$ and Alvin Y Liu ${ }^{7,8}$

${ }^{1}$ Department of Pathology, University of Washington, Seattle, WA, USA; ${ }^{2}$ Institute for Systems Biology, Seattle, WA, USA; ${ }^{3}$ Department of Genome Sciences, University of Washington, Seattle, WA, USA; ${ }^{4}$ Section of Human Biology, Fred Hutchinson Cancer Research Center, Seattle, WA, USA; ${ }^{5}$ MacroGenics, Seattle, WA, USA; ${ }^{6}$ Biological Science Division and Environmental Molecular Sciences Laboratory, Pacific Northwest National Laboratory, Richland, WA, USA; ${ }^{7}$ Department of Urology, University of Washington, Seattle, WA, USA and ${ }^{8}$ Institute for Stem Cell and Regenerative Medicine, University of Washington, Seattle, WA, USA

A by-product in the processing of prostate tissue for cell sorting by collagenase digestion is the media supernatant that remains after the cells are harvested. These supernatants contain proteins made by the cells within the tissue. Quantitative proteomic analysis of $\mathrm{N}$-glycosylated proteins detected an increased amount of CD90/THY1 in cancer supernatants compared with non-cancer supernatants. Immunohistochemistry showed that in all carcinomas, regardless of Gleason grade, a layer of CD90-positive stromal fibroblastic cells, $\sim 5$ to 10 cells deep, was localized to tumor glands. In contrast, a no more than 1-cell wide girth of CD90-positive stromal cells was found around benign glands. The increased number of CD90-positive stromal cells in cancer correlated with overexpression of CD90 mRNA detected by gene expression analysis of stromal cells obtained by laser-capture microdissection. There is increasing evidence that cancer-associated stroma has a function in both tumor progression and carcinogenesis. Most experiments to identify cancer biomarkers have focused on the cancer cells. CD90, being a marker for prostate cancer-associated stroma, might be a potential biomarker for this cancer. A non-invasive test could be provided by a urine test. Proteomic analysis of urine from patients with prostate cancer identified CD90; conversely, CD90 was not detected in the urine of post-prostatectomy patients. Furthermore, this urinary CD90 protein was a variant $C D 90$ protein not known to be expressed by such cells as lymphocytes that express CD90. These CD90 results were obtained from $\sim 90$ cases consisting of proteomic analysis of tissue and urine, immunohistochemistry, western blot analysis of tissue media, flow cytometry of cells from digested tissue, and reverse transcriptase polymerase chain reaction analysis of isolated stromal cells.

Modern Pathology (2010) 23, 1346-1356; doi:10.1038/modpathol.2010.122; published online 18 June 2010

Keywords: cancer; CD90 variant; glycoproteins; prostate; proteomics; stroma; urine analysis

Correspondence: Dr LD True, MD, Department of Pathology, University of Washington, Box 356100, 1959 NE Pacific Street, NE-110, Seattle, WA 98195-6100, USA.

E-mail: ltrue@uw.edu

${ }^{9}$ Current address: Department of Pathology, Johns Hopkins University, Baltimore, MD, USA.

${ }^{10}$ Current address: Division of Biotechnology, Dalian Institute of Chemical Physics, Chinese Academy of Sciences, Dalian, China.

${ }^{11}$ Current address: Proteomics Resources, University of Washington, Seattle, WA, USA.

${ }^{12}$ Current address: XORI, Seattle, WA, USA.

Received 28 November 2009; revised 7 May 2010; accepted 10 May 2010; published online 18 June 2010
Stromal cells have an important function in the structural and functional properties of epithelium in many organs. In normal development of the genitourinary system, stromal cells have a critical function in directing epithelial differentiation. Cunha et al. demonstrated in experiments with rodents that the gender of origin of stromal mesenchyme cells determined the differentiation of juxtaposed, coimplanted epithelial cells. Specifically, male mesenchyme directed coimplanted female epithelium to form androgen receptor-expressing glands. 
Conversely, female mesenchyme directed coimplanted male epithelium to form estrogen receptorexpressing vaginal epithelium-like, stratified squamous epithelium. ${ }^{1}$

Extending these concepts to human prostate carcinoma, these investigators showed that prostate cancer-associated fibroblasts could induce proliferation and malignant transformation of cultured prostate epithelial cells under androgen stimulation, in contrast to non-cancer-associated fibroblasts. ${ }^{1}$ Furthermore, there is evidence that prostate cancer-associated stroma 'drives' tumor progression, mediated by growth factors of stromal cells. ${ }^{2}$ Similar dependence of proliferation and other cancerassociated properties on stromal cells has been shown in other tissues, such as the breast. However, little is known of the molecular details of these processes. Neither the molecular phenotype of prostate cancer-associated fibroblasts nor the molecular mediators of the stromal/epithelial interaction have been characterized.

Identifying cell membrane molecules that characterize and distinguish different parenchymal cell types is of biological importance. These molecules are critical for a range of functional properties including cell signaling, receptor activation, and cell adhesion. These molecules can also be used as markers to identify and sort defined cell types, and to serve as cell-specific targets for therapy. Using monoclonal antibodies specific to the cell membrane cluster designation (CD) molecules, we have characterized the parenchymal cells of the prostate, and identified CD molecules that are uniquely expressed by the various parenchymal cells of the prostate. ${ }^{3}$ Furthermore, we have found that primary prostate carcinomas that differ in their CD phenotype have apparently different malignant potential. ${ }^{4}$ We now extend these studies to characterizing the phenotype of cancer-associated stroma with the goal of better understanding the molecular aspects of stroma/cancer cell interaction. One practical application is in determining whether the presence of these cancer-associated stromal cells could be used for cancer detection.

\section{Materials and methods}

This study is approved by our institutional review board.

\section{Analysis of Differentially Expressed Proteins in Cancer}

Glycopeptide-capture proteomic analysis of tissue preparations

Matched cancer and non-cancer tissue specimens were dissected from surgically resected glands. The tissue samples were minced and digested by collagenase in serum-free RPMI1640 media supplemented with $10^{-8} \mathrm{M}$ dihydrotestosterone.
The cell-free post-digestion supernatants of two pairs of cancer/non-cancer cases were used for identification of differentially expressed N-linked glycoproteins by glycopeptide-capture tandem mass spectrometry. ${ }^{5-7}$ This method involved: (1) glycoprotein oxidation with periodate to convert the cis-diol of carbohydrate to aldehyde; (2) coupling of the generated aldehyde to hydrazide on solid support; (3) proteolysis of immobilized glycoproteins by trypsin; (4) isotopic labeling of cancer and non-cancer samples; (5) release of labeled glycopeptides by peptide- $N$-glycosidase (PNGase) F; and (6) analysis of peptides by microcapillary liquid chromatography-electrospray ionization-tandem mass spectrometry ( $\mu \mathrm{LC}$-ESI-MS/MS) or $\mu \mathrm{LC}$ followed by matrix-assisted laser desorption/ionization tandem mass spectrometry (MALDI-MS/MS). The proteins thus identified were predominantly classed as extracellular. ${ }^{8}$ HPLC-grade reagents purchased from Fisher Scientific (Pittsburgh, PA, USA) were used for chromatography procedures. PNGase $F$ was purchased from New England Biolabs (Beverly, MA, USA) and hydrazide resin from Bio-Rad (Hercules, CA, USA). All other chemicals used in this study were purchased from Sigma (St Louis, MO, USA). Acquired MS/MS spectra were searched against the International Protein Index human protein database (version 2.28, containing 40110 entries) using SEQUEST software. ${ }^{9}$ The database search tool was set to include the following amino acids: carboxymethylated cysteines, oxidized methionines, and enzyme-catalyzed conversion of Asn to Asp at the site of carbohydrate attachment, and to accept at least one tryptic end and up to two missed cleavages. The mass tolerance for precursor ions was 3.0 Da and that for fragment ions was 0.6 Da. The default values were used for the other parameters in the database search. Search results were statistically analyzed using PEPTIDEPROPHET, which effectively computes a probability (on a scale of 0-1) for the likelihood of each identification being correct in a data-dependent manner. ${ }^{10} \mathrm{~A}$ minimum PEPTIDEPROPHET probability score filter of 0.9 was used to remove low-probability peptides. As N-linked glycosylation occurs at a consensus Asn-X-Ser/Thr sequon, ${ }^{11}$ the remaining peptide sequences were additionally filtered to remove non-motif-containing peptides.

\section{Immunohistochemistry}

Primary prostate cancers from radical prostatectomy specimens comprised the tissue for the immunohistochemistry studies. On receipt of the radical prostatectomy specimen, 3-mm thick transverse sections were made after inking the exterior surface. Tissue blocks from the posterior aspect of each alternate transverse section were embedded in Optimal Cutting Temperature (OCT, Tissue-Tek, Torrance, CA, USA) and snap frozen in isopentane that had been pre-cooled in liquid nitrogen. Frozen 
sections of these blocks provided a template for locating the cancer.

Frozen sections from $\sim 60$ cases of OCT-embedded tissue were cut onto charged slides, fixed for $10 \mathrm{~min}$ in cold acetone and kept refrigerated at $-20^{\circ} \mathrm{C}$ until use. In staining with a monoclonal CD90 antibody (clone 5E10, BD-PharMingen, San Diego, CA, USA), sections were incubated sequentially in solutions of $0.03 \%$ hydrogen peroxide, $0.5 \%$ albumin in phosphate-buffered saline (PBS), primary antibody $(5 \mu \mathrm{g} / \mathrm{ml})$, secondary biotinylated anti-mouse IgG (BA-2000, Vector Labs, Burlingame, CA, USA) with interval washes in PBS. Reaction product was detected by incubating sections in solution of $0.05 \%$ diaminobenzidine (Invitrogen, Carlsbad, CA, USA). The sections were counterstained with hematoxylin. Details of the method are available on the web (http://scgap.systemsbiology. net/resources/protocols.php).

\section{Western blot analysis}

As the tissue-digestion media supernatants contained large amounts of added collagenase, the amount used for analysis was based on grams of tissue $/ \mathrm{ml}$ of media instead of protein concentration. Samples were electrophoresed on $4-20 \%$ gradient SDS-polyacrylamide gels and electrotransferred to membrane. After treatment in 5\% non-fat milk, the membrane was probed with CD90 antibody (1:500) for $60 \mathrm{~min}$, followed by horseradish peroxidase conjugated anti-mouse IgG. Detection was by using Luminol (Santa Cruz Biotechnology, Santa Cruz, CA, USA). Prostate-specific antigen (PSA) antibody (1:1000, clone A67-B/E3, Santa Cruz Biotechnology) was used for sample loading control. Thirty cancer specimens were probed by western blot and the result for seven is shown in the data figure. Tissue inhibitor of metalloproteinase 1 (TIMP1) antibody (clone 7-6C1, Chemicon, Temecula, CA, USA) was used at 1:1000. TIMP1, a luminal secreted protein, is absent in cancer tissue. ${ }^{6}$

\section{Cancer-Associated Stromal Cells}

Flow analysis of tumor tissue

A tumor sample, 98-366, was dissected from the prostate using a stained section as guide. It was digested with collagenase in media with $10^{-8} \mathrm{M}$ dihydrotestosterone. The resultant single-cell suspension was analyzed for the percentage of CD57, CD107b, CD90, and CD49a cells by FACS as described. ${ }^{12}$ CD57 and CD107b mark luminal epithelial cells; CD90 and CD49a mark stromal cells. ${ }^{3}$

\section{Laser-capture microdissection}

In all, $5 \mu \mathrm{m}$-thick sections were cut from OCTembedded, frozen blocks of prostate tissue from 10 radical prostatectomy specimens. The sections were briefly stained ( $\sim 5 \mathrm{~s}$ ) with RNase-free hematoxylin using HistoGene Staining Solution (Arcturus
Engineering, Mountain View, CA, USA), sequentially dehydrated through a gradient of ethanol into xylene, and allowed to air dry in low-humidity atmosphere for $<15 \mathrm{~min}$. Using Arcturus Pixcell II laser-capture microscopy, 2000 stromal cells were obtained within 20 min from each section. ${ }^{13}$ Both cancer-associated stromal cells (within 10 cell layers of cancer) and benign gland-associated stromal cells (within 10 cell layers of benign glands) were separately microdissected from each of these 10 blocks. Images of each laser-capture microdissection were taken for documentation.

\section{RNA amplification and gene expression analysis} of stromal cells

Captured cells were lysed in Arcturus RNA extraction buffer. RNA was isolated using Arcturus PicoPure RNA isolation, and the samples were treated with RNase-free DNase I Set (Qiagen, Valencia, CA, USA). T7-based RNA amplification was performed using MessageAmp Amplification (Ambion, Austin, TX, USA). The first-round amplified RNA (aRNA) was used as a template for a second round of amplification to yield 50-150 $\mu \mathrm{g}$ aRNA.

A measure of $2 \mu \mathrm{g}$ aRNA was reverse transcribed using SuperScript II (Invitrogen). The reaction product was then hydrolyzed in $0.2 \mathrm{M} \mathrm{NaOH}$, $0.1 \mathrm{M}$ EDTA for $15 \mathrm{~min}$ at $65^{\circ} \mathrm{C}$ and neutralized with $0.33 \mathrm{M}$ Tris-HCl, pH 7.4. The cDNA was purified with Qiagen PCR clean-up column chromatography. Relative quantification of gene expression by PCR (40 cycles of $95^{\circ} \mathrm{C}, 30 \mathrm{~s} ; 60^{\circ} \mathrm{C}, 30 \mathrm{~s} ; 72^{\circ} \mathrm{C}, 30 \mathrm{~s}$ ) was done on 7700 Sequence Detector using SYBR Green Master (ABI, Foster City, CA, USA) and genespecific primers. Sample results were normalized to the cycle threshold value obtained in the exponential amplification of the housekeeping gene glyceraldehyde-3-phosphate dehydrogenase (GAPDH). Expression levels of CD90 were calculated, and values were reported as the ratio of gene expression in cancer-associated stromal cells to noncancer tissue stromal cells. The error of the mean from three experiments was represented by error bars. Control reactions with RNA or water as template were negative. Amplification of a singlePCR product per reaction was monitored by the generation of a single-dissociation curve. The sequences of primers used were: GAPDH-5, CCTCA ACGACCACTTTGTCA and GAPDH-3, TTACTCCTT GGAGGCCATGT; CD90-5, TCAGGAAATGGCTTT TCCCA and CD90-3, TCCTCAATGAGATGCCATA AGCT. The CD90 primers match to sequences $100 \mathrm{bp}$ apart in the $3^{\prime}$ untranslated region (UTR) of the mRNA near the poly-A sequence.

\section{Urine Analysis}

Proteomic analysis of urine

Isotope-coded affinity tag (ICAT) labeling and MS/ MS was used to identify differentially expressed 
proteins in urine samples collected from consented pre-op(erative) and post-op patients. The urine samples were collected and stored overnight at $4^{\circ} \mathrm{C}$ before use. Urinary proteins as assessed by silver staining of polyacrylamide gels were found to be stable for at least 1 week at that temperature (for long term, the samples were stored at $-80^{\circ} \mathrm{C}$ ). Western blot analysis for the male-specific prostatic acid phosphatase (PAP/ACPP) showed a single-discrete band (data not shown). A measure of $15 \mathrm{ml}$ of urine were concentrated with spin filters (3-kDa cutoff) to yield protein concentrations ranging from 1.4 to $3.7 \mathrm{mg} / \mathrm{ml}$. For ICAT labeling, ${ }^{14,15} 200-300 \mu \mathrm{g}$ of concentrated proteins from each sample were used. After tryptic digestion, peptides were separated into 10 cation exchange fractions, and the labeled (isotope d0 or d9) Cys-containing peptides were isolated by avidin affinity chromatography. The peptides were analyzed by LC-MS/MS and the proteins were identified from the spectral data as described above. High abundance urinary proteins, as assessed by silver-stained polyacrylamide gels, were identified by in-gel-digest followed by LC-MS/MS. ${ }^{16}$

In a different approach, urine samples were desalted and concentrated using Amicon Ultra-15 centrifugal filter MWCO3000 (Millipore, Billerica, MA, USA). Protein concentration was determined by BCA Protein Assay (Thermo Scientific, Rockford, IL, USA). An aliquot of concentrated protein sample was denatured in $50 \%$ trifluoroethanol at $60^{\circ} \mathrm{C}$ for $2 \mathrm{~h}$ with gentle shaking, and then reduced with $2 \mathrm{mM}$ DTT at $37^{\circ} \mathrm{C}$ for $1 \mathrm{~h}$. Samples were diluted fivefold with $50 \mathrm{mM} \mathrm{NH}_{4} \mathrm{HCO}_{3}$ before digestion with trypsin (trypsin:protein 1:50 w/w) at $37^{\circ} \mathrm{C}$ for $3 \mathrm{~h}$. After speed-vac, peptides were reconstituted in $50 \mathrm{mM} \mathrm{NH}_{4} \mathrm{HCO}_{3}$ for LC-MS analysis. Urine peptide samples $(0.5 \mu \mathrm{g} / \mu \mathrm{l})$ were analyzed by capillary-LCselected reaction monitoring (SRM)-MS on a TSQ Quantum Ultra triple quadrupole mass spectrometer (ThermoFisher Scientific, Waltham, MA, USA) coupled with an in-house automated four-column reverse-phase HPLC system (Pacific Northwest National Laboratory). The mobile phase consisted of $0.1 \%$ formic acid in $\mathrm{H}_{2} \mathrm{O}$ (solution $\mathrm{A}$ ) and $0.1 \%$ formic acid in acetonitrile (solution B). A measure of $5 \mu \mathrm{l}$ of peptides was loaded onto the column, and the mobile phase was maintained at $100 \%$ solution A for $20 \mathrm{~min}$. The mobile phase was increased with solution $\mathrm{B}$ exponentially over $80 \mathrm{~min}$ to a final concentration of $70 \%$. The reverse-phase capillary column was $75 \mu \mathrm{m}$ i.d. and $70 \mathrm{~cm}$ long fused silica (Polymicro Technologies, Phoenix, AZ, USA) slurry packed with $3 \mu \mathrm{m}$ C18 Jupiter-bonded particles with $300 \AA$ pores (Phenomenex, Torrence, CA, USA). Q1 and Q3 resolution was set at 0.7 FWHM and Q2 collision gas pressure was $1.5 \mathrm{mTorr}$. Scan width and time were optimized as $0.001 \mathrm{~m} / \mathrm{z}$ and $10 \mathrm{~ms}$, respectively. Peptides for CD90 (and others) detected initially by LC-MS/MS of the same urine sample were selected for SRM analysis with the top seven most abundant/intense fragments being monitored.

\section{Results}

\section{Prostate Tumor Production of CD90 Protein}

The collagenase tissue-digestion media preparations were used for proteomics on the assumption that during enzymatic treatment, cells remain metabolically active and continually secrete cell type-specific protein products. Earlier, we have shown by western blot analysis that there was minimal protein degradation, and the prostatic proteins of PSA/KLK3, ACPP and zinc- $\alpha 2$-glycoprotein (AZGP1) were readily detected.6,17 Our methodology specifically targeted N-linked glycoproteins, and identified a formerly $N$-glycopeptide, LDCRHENTSSSPIQYEFSLTR (mass 2541.15, N-glycosylation motif underlined), from CD90 with a quantitative increase in cancer. Another CD90 N-glycopeptide observed from the same glycosite was HENTSSSPIQYEFSLTR (1994.92, minus the N-terminal LDCR of the first). A different N-glycosite, DEGTYTCALHHSGHSPPIS SQNVTVLR (2905.37), was also identified from CD90. Table 1 lists 10 proteins quantified in the

Table 1 CP vs NP N-glycoproteomes

\begin{tabular}{lcll}
\hline Protein & \#1 Ratio & \#2 Ratio & Annotation \\
\hline IPI00024284 & 0.19 & 0.61 & Basement membrane-specific heparan sulfate proteoglycan core protein \\
IPI00289983 & 0.22 & 0.06 & Prostatic acid phosphatase \\
IPI00178017 & 0.24 & 0.54 & Angiotensin 1 converting enzyme isoform 3 \\
IPI00022792 & 0.36 & 0.78 & Microfibril-associated glycoprotein 4 \\
IPI00023673 & 0.38 & 0.81 & MAC-2 binding protein \\
IPI00383517 & 0.80 & 0.49 & Prostate-specific antigen \\
IPI00298281 & 1.39 & 1.87 & Laminin $\gamma-1$ chain \\
IPI00329573 & 1.94 & 1.36 & Long splice isoform of Q99715 collagen $\alpha$ 1 (XII) chain \\
IPI00022892 & 5.04 & 4.49 & CD90, Thy-1 membrane glycoprotein \\
IPI00328113 & 7.18 & 1.60 & Fibrillin 1
\end{tabular}

The ratio is the relative abundance of the detected isotopic peptides in cancer compared with non-cancer. The results from two pairs of samples (\#1 and \#2) are shown. CP, prostate cancer tissue; NP, non-cancer prostate tissue. 
two matched cancer and non-cancer specimens, and CD90 showed an increase of 5- and 4.5-fold, respectively, in the ratio of its signature peptide in cancer over non-cancer. The experimental data for CD90 is archived in https://db.systemsbiology.net/ sbeams/cgi/Glycopeptide/Glyco_prediction.cgi. With the exception of CD90, most of the identified glycoproteins (eg PSA, Table 1; TIMP1, not shown) in these collagenase-digestion supernatants showed a decrease in cancer.

Western blot analysis confirmed the glycopeptidecapture result. For each cancer specimen analyzed, there was an increased amount of CD90 protein ( $\sim 27 \mathrm{kDa}$ ) compared with non-cancer (Figure 1). As shown earlier, these specimens were low in TIMP1 $(30 \mathrm{kDa})$, which is synthesized by luminal cells in non-cancer but not by cancer cells. ${ }^{6}$ PSA was detected in both cancer and non-cancer specimens. ${ }^{17}$ A tumor specimen protein profile can thus be characterized as CD90 ${ }^{\text {hi }}$ TIMP $1^{\text {lo }}$ and a non-cancer

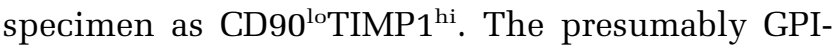
anchored CD90 appeared to be released from the cells.

Immunohistochemical analysis of prostate cancer samples showed distinct and differential overexpression of CD90 in cancer-associated stroma compared with non-cancer tissue stroma. The increased CD90 protein level (based on the increased number of stained stromal cells, Figure 2) was seen in all 30 peripheral zone and transition zone tumors studied. No difference was observed in cancers of different Gleason grade. The image results for 43 radical prostatectomy specimens are available for query and download at https://db.systemsbiology.net/sbeams/ cgi/Immunostain/main.cgi?set_current_project_id = 274 and https://db.systemsbiology.net/sbeams/cgi/ Immunostain/SummarizeStains. The increased number of stained stromal cells, which did not extend beyond 10 stromal cells from the tumor cells,

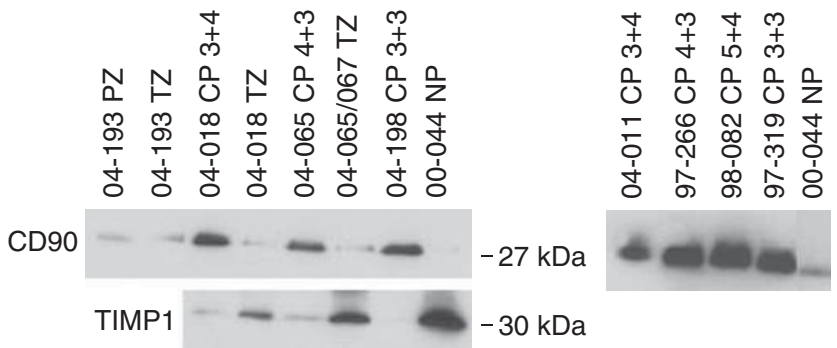

Figure 1 CD90 glycoprotein in cancer tissue. Western blot analysis of tissue-digestion media detected a diffuse CD90 band at $\sim 27 \mathrm{kDa}$, which is larger than the calculated molecular weight of CD90 ( 16 kDa) based on the amino-acid sequence. Cancer is characterized as $\mathrm{CD}^{\mathrm{hi}}{ }^{\mathrm{hi}}$ and non-cancer as $\mathrm{CD} 90^{\mathrm{lo}}$. Both peripheral zone and transition zone cancer show increased CD90 expression compared with non-cancer. Expression level does not seem to vary with Gleason scores. Note that the Gleason score applies to the case not necessarily to the specimens obtained for collagenase digestion. The $30-\mathrm{kDa}$ TIMP1 can be used to characterize non-cancer specimens as TIMP1 is synthesized by luminal cells and not cancer cells. PZ, peripheral zone; TZ, transition zone. was localized around the cancer glands. For a typical benign gland, there was some CD90 staining just outside the basal epithelium, but it was weak elsewhere in the stroma. Flow analysis of a cancer specimen showed a high percentage of $\mathrm{CD}^{+}{ }^{+}$cells (data not shown; sorting of CD90 ${ }^{+}$cells was recently reported in Pascal et al. $\left.{ }^{17}\right)$. An equal percentage of $\mathrm{CD} 7^{+}$and CD107b ${ }^{+}$cancer cells was found.

In 8 of 10 laser-captured stromal cell sample pairs, an increase in CD90 transcript level in cancer was obtained by qRT-PCR analysis using primers to the 3' UTR (Figure 3). The estimated fold increase in cancer over non-cancer ranged from 1.2 to 7.6. There was no increase detected in two cases. This increase in CD90 transcript corresponded with the increase in CD90 protein detected in tumor tissue samples.

\section{Detection of CD90 Protein in Urine}

As CD90 might be released from cells, voided urine from cancer patients might contain detectable amounts of CD90. To assess whether CD90 could be a viable prostate cancer biomarker, urine was collected from patients and healthy donors, and analyzed by proteomics methods. The collected patient urine samples were first shown by western blot analysis to contain such prostate-abundant proteins as ACPP and AZGP1 (data not shown). The major urinary proteins identified through in-gel digestion included uromodulin, MAC-2-binding protein, albumin, immunoglobulin $\gamma 1$, VIP-36, AMBP, APOD, prothrombin, immunoglobulin $\kappa$, prostaglandin-h2-isomerase (Figure 4). Proteomic analysis of urine samples from prostate cancer patients $(n=3$, labeled with d9) identified a CD90 proteotypic peptide, VTSLTAC ${ }^{*}$ LVDQSLR $\left(\mathrm{C}^{*}\right.$ to indicate ICAT-labeled Cys residue), in the pre-op samples but not in the post-op samples (labeled with d0, Figure 5). At the same time, a drop in PSA (as represented by peptide KLQC ${ }^{*}$ VDLHVISNDVC* AQVHPQK) after surgical removal of the gland was seen in the post-op urine. The ICAT method did not specifically target secreted proteins; the relative fractions of the identified proteins $(n=285)$ was: $28 \%$ secreted, $10 \%$ extracellular, $25 \%$ transmembrane, 24\% intracellular, and $13 \%$ unknown. $\mathrm{PSP}_{94} / \mathrm{MSMB}$, a Cys-rich prostate secretory protein, showed the highest $\mathrm{d} 9 / \mathrm{d} 0$ ratio at $>10$.

Analyzed by a different method, a CD90 peptide, WVGGPCAAGP, detected in a pre-op urine (Figure 6) matched the N-terminus of a variant CD90 protein (NCBI accession number BAD92446) encoded by a cDNA clone found in a human library. This variant differs from the T-cell CD90 at both the $\mathrm{N}$ - and C-termini. The two CD90 proteins appear to arise from differential splicing (Figure 7). In the same analysis, a peptide for the breast cancer membrane protein 11 (BCMP11) protein 

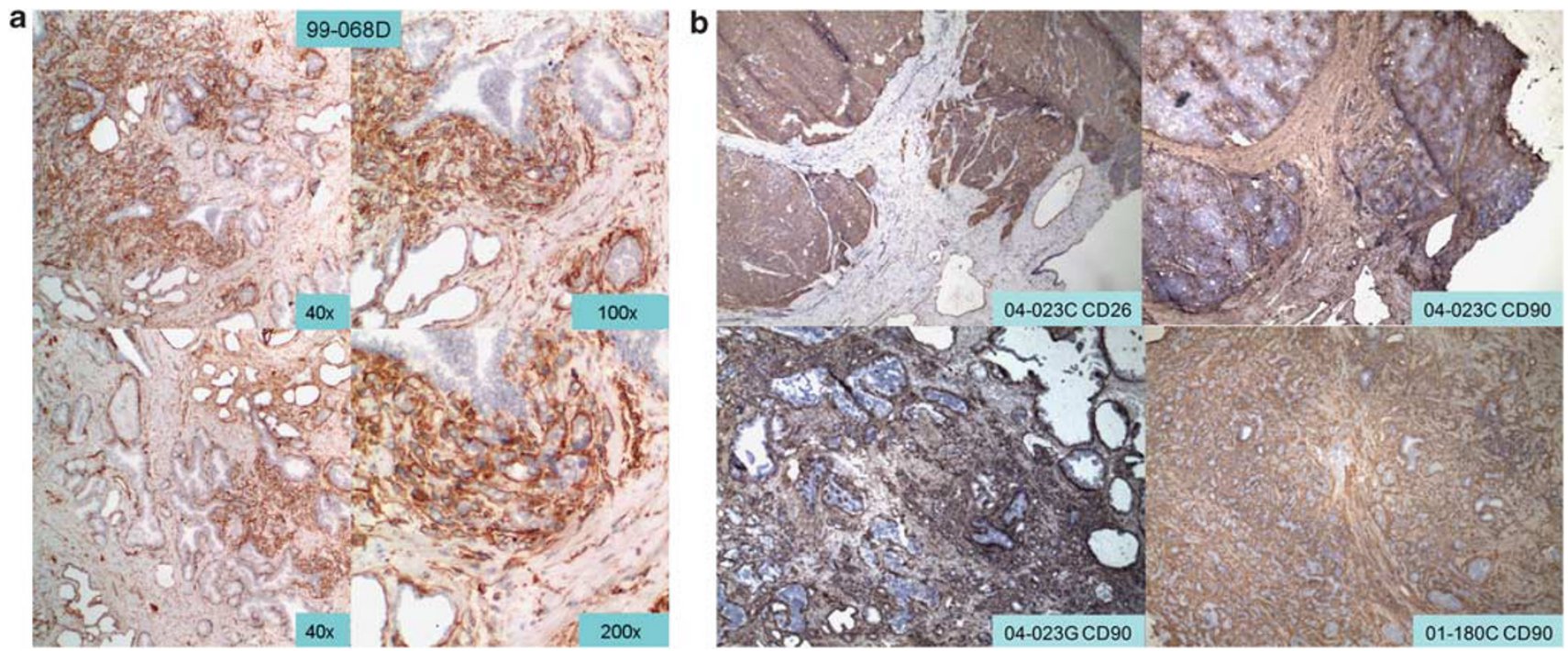

Figure 2 CD90 staining of cancer-associated stromal cells. (a) CD90 staining of tissue specimen 99-068D shows increased reactivity localized to the tumor foci. Staining in non-cancer is restricted to a cell layer adjacent to the basal epithelium. Blood vessels are also positive for CD90. Original magnifications are indicated in the individual photomicrographs. (b) The transition zone cancer, 04-023, contained both tissue without benign glands (top panels) and cancer-rich tissue with scattered benign glands (bottom left). The tumor cells in 04-023C are stained by CD26 (top left). 01-180C contained a large tumor focus with crowded glands. Original magnification $\times 40$.

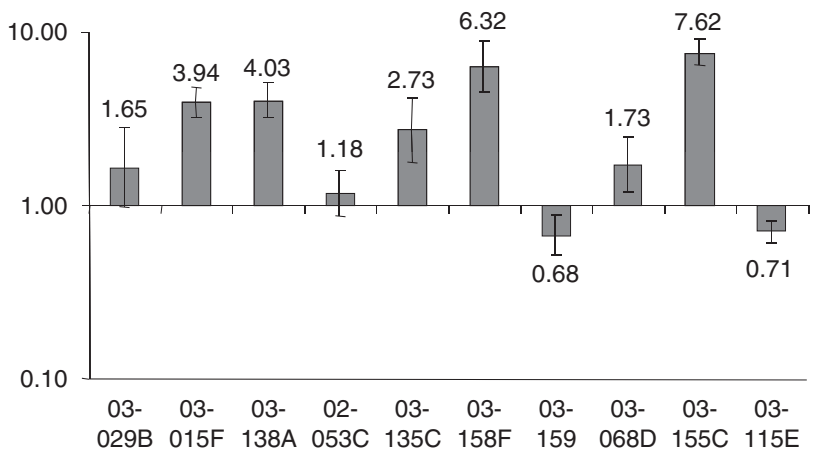

Figure 3 CD90 expression analysis in laser-captured stromal cells. Matched cancer and non-cancer specimens were prepared from cases listed on the $x$-axis. The fold differences in CD90 transcript expression for the laser-captured stromal cell samples are indicated on the $y$-axis in the range of 0.1 - to 10 -fold cancer over non-cancer. Increased transcript was seen in 8 of 10 cancerassociated stromal cell samples.

was also detected (Figure 6). BCMP11 (also known as AGR3) was one of the genes encoding secreted/ extracellular proteins found upregulated in CD26 ${ }^{+}$ prostate cancer cells compared with $\mathrm{CD}_{2} 6^{+}$luminal cells. ${ }^{18}$

\section{Discussion}

CD90 was first identified in the thymus as a T-cell maturation and differentiation marker (http:// www.ncbi.nlm.nih.gov/prow/). Although known to be expressed in primitive hematopoietic progenitor cells, thymocytes and fibroblasts, CD90 has an unknown function in prostate biology. Studies have reported differential expression of CD90 in fibroblasts from many tissues including lung, myometrium, and orbit. ${ }^{19}$ CD90 expression distinguishes fibroblasts by several functional properties: (1) their differentiation potential-CD90 ${ }^{+}$myofibroblastic vs $\mathrm{CD}^{-} 0^{-}$lipofibroblastic ${ }^{20}$ and (2) their responsiveness to growth factor signaling- $\mathrm{CD}^{-}{ }^{-}$fibroblasts respond to PDGF, IL-1 $\beta$, IL-4 with increased TGF- $\beta$ activity, Smad3 phosphorylation, expression of $\alpha$-smooth muscle actin (SMA) and fibronectin; whereas $\mathrm{CD}^{+}{ }^{+}$fibroblasts resist stimulation by these factors. ${ }^{21}$ In addition, fibroblast activation of CD90 expression is dependent on participation of the CD40-ligand pathway and TGF- $\beta$ signaling. ${ }^{22,23}$ It is possible that $\mathrm{CD}^{+} 0^{+}$prostate stromal fibroblastic cells might also behave differently compared with non-cancer-associated CD90 ${ }^{\text {lo }}$ stromal cells.

In prostate cancer, there could be defects in stromal/epithelial interaction. The stroma is populated predominantly by smooth muscle cells (which are desmin ${ }^{+}$, caldesmon ${ }^{+}$, and $\alpha \mathrm{SMA}^{+}$) with few myofibroblasts $\left(\alpha \mathrm{SMA}^{+}\right.$, vimentin $\left.{ }^{+}\right)$and fibroblasts (vimentin ${ }^{+}$). In cancer, the proportion of smooth muscle cells is decreased, whereas the amount of collagen fibers is increased. ${ }^{17,24,25}$ There is also loss of androgen receptor ${ }^{26}$ and calponin ${ }^{27}$ expression. Does the emergence of CD90 ${ }^{+}$cells represent a shift in stromal cell differentiation? The stromal cells are characterized as CD49a ${ }^{+}, \mathrm{CD}^{2} 6^{+}$, and CD90 $0^{\mathrm{lo}}$ in benign tissue. ${ }^{28}$ When prostate stromal cells are cultured in vitro, the resultant population of cells was found by flow cytometry to have increased representation of $\mathrm{CD}^{+}{ }^{+}$cells and decreased representation of $\mathrm{CD}^{2} 6^{+}$cells. ${ }^{29}$ Transcriptomes of 
$\mathrm{CD}^{+}$cells sorted from tumor specimens have recently been reported by $\mathrm{us}^{17}$ which showed cancer-associated stromal cells had lower expression of smooth muscle cell genes. The fibroblasts of melanoma also stain strongly for CD90. ${ }^{30}$

Quantitative proteomic analysis of tissue samples found increased CD90 glycopeptide in cancer vs non-cancer. Proteins with increased cancer expression were a minority, perhaps reflecting the fact that

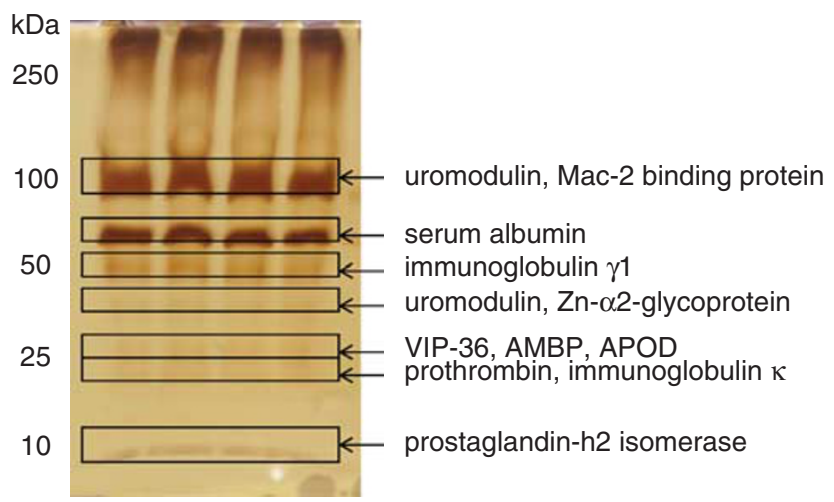

Figure 4 Major human urinary proteins. Polyacrylamide gelresolved urinary proteins from four healthy donor specimens are shown. Identification was achieved by in-gel digestion and mass spectrometry. The band intensities show that there is no significant variation between samples. tumor cells are non-functional cell types. TIMP1 is an example of a highly expressed secreted proteins showing cancer non-expression, which we have reported earlier. ${ }^{6}$ The presence of CD90 in the media could be due to cell shedding, secretion, or enzymatic release. Because of its elevated expression, CD90 seems to be a good cancer biomarker candidate, especially given the fact that not many candidates with increased expression specific to cancer cells have been detected in these proteomeprofiling experiments. Other prostate cancer upregulated genes like $\alpha$-methylacyl-CoA racemase is localized to peroxisomes and mitochondria, hepsin is a single-pass type II membrane serine protease, prostate cancer antigen (PCA3) is a non-proteincoding transcript. As extracellular CD90 could be found in tissue collagenase-digestion supernatant, we wondered whether CD90 might be detected in the urine of cancer patients. A urine test would represent a desirable non-invasive means of cancer detection. Accordingly, a CD90 proteotypic peptide was found to be of higher abundance in urine samples from three patients compared with urine samples of post-op (and healthy) men. Although we anticipate investigating correlation of CD90 expression with clinical outcome, such an investigation is a project planned for the time when we have enough cases for statistical analysis, along with IRB approval to obtain outcomes data.

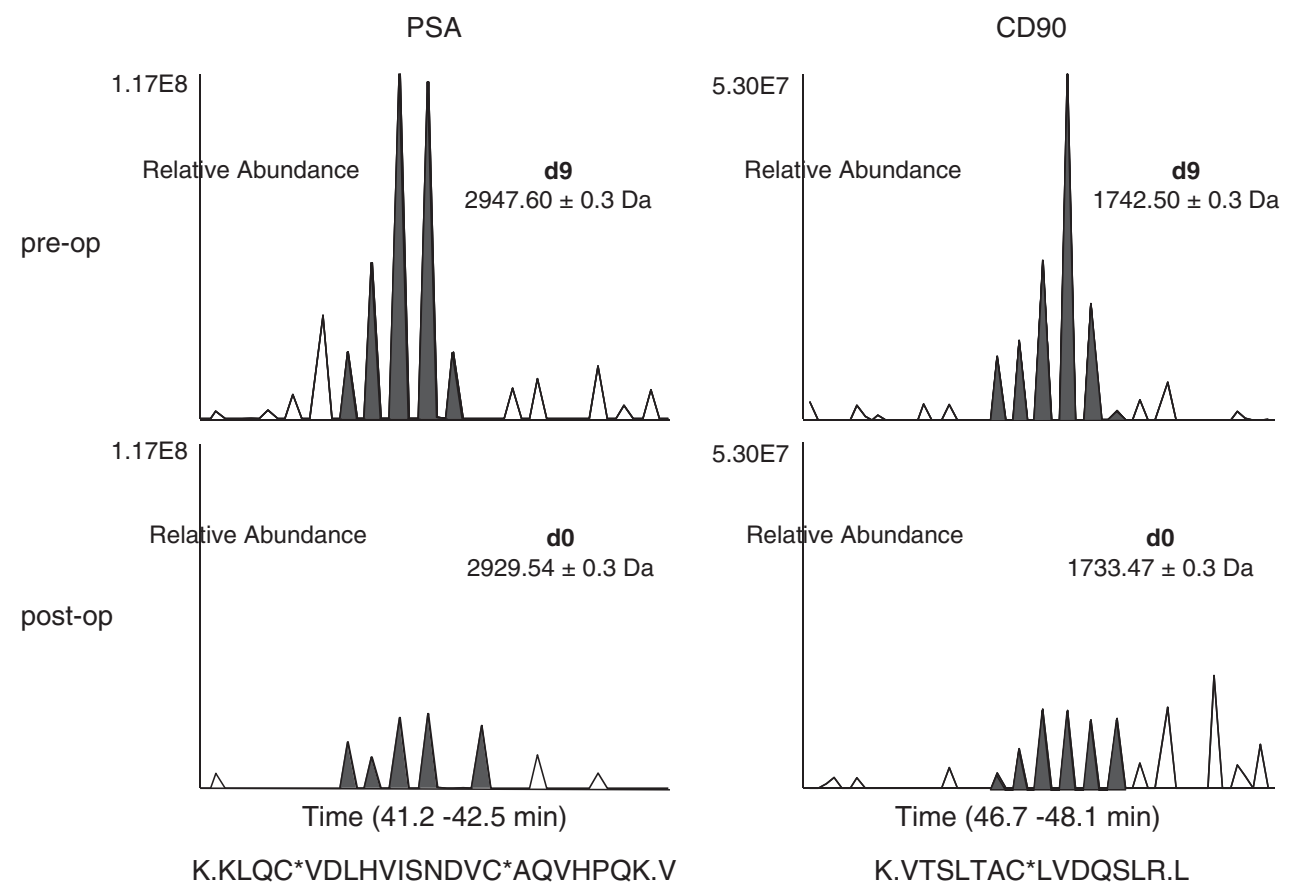

Figure 5 CD90 in urine. Single-ion traces of the mass from heavy (d9, pre-op urine) and light (d0, post-op urine) ICAT-labeled peptides indicate that PSA and CD90 are detectable in prostate cancer patient urine. The elution profiles (single-ion traces of the peptide mass over time) of heavy-labeled peptide masses and those of the corresponding light-labeled peptide masses are highlighted. The detected peptide sequences are shown below the traces. The weight difference of 18 in PSA is due to the two-labeled Cys residues. Both PSA (more abundant than CD90, y-axis) and CD90 show a similar trend wherein both proteins are present in the pre-op sample but below detection limit in the post-op sample. 
CD90/THY1

WVGGPCAAGP $(\mathrm{m} / \mathrm{z} 457.71,2+)$

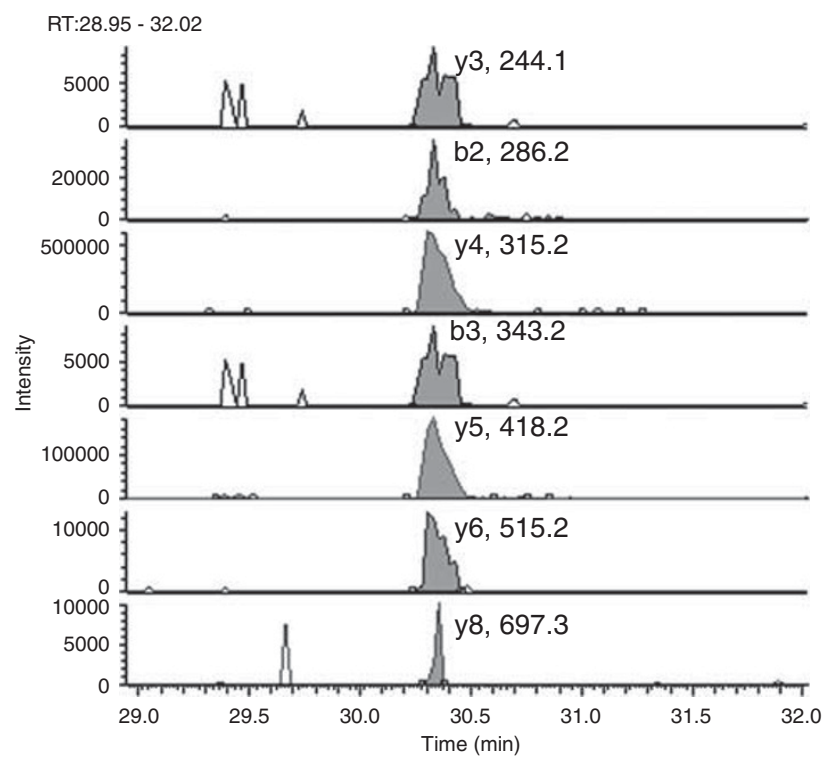

BCMP11

DLPLLIENMK ( $\mathrm{m} / \mathrm{z} 593.33,2+)$

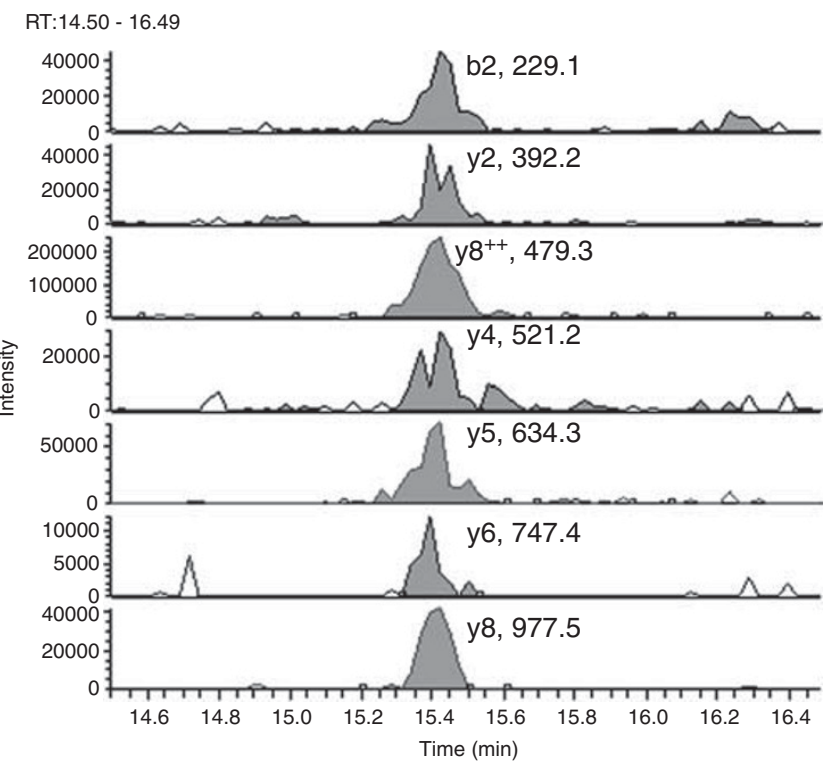

Figure 6 CD90 variant. The panel shows LC-SRM-MS detection of seven MS/MS fragments of a peptide specific to the CD90 variant protein (BAD92446) encoded by a CD90 cDNA clone (AB209209) in a pre-op urine sample. In the same sample, a peptide of the cancer upregulated BCMP11 was also detected.

However, as CD90 is overexpressed in virtually all tumors, it may not have prognostic value like differentially expressed CD10. ${ }^{4}$ One testable prediction is that the amount of urinary CD90 could correlate with tumor size.

The detection of a variant CD90 in urine of a cancer patient suggests that cancer-associated stromal cells produce this variant. This variant was previously identified from a human protein-coding cDNA project (RIKEN), and there are no documented studies on the encoded protein. The variant encoding mRNA was not identified in a T-cell cDNA library from which CD90 was first cloned. ${ }^{31}$ Thus, it is possible that stromal fibromuscular cells and lymphocytes express a different CD90 protein. The variant (stromal) version does not contain a hydrophobic transmembrane segment at its C-terminus. An initiating Met codon has not been identified for this variant. The N-terminal WVGGPCAAGP sequence is also found at position 213-222 of the chimpanzee (Pan troglodytes) Thy1 protein (XP_508814) inferred from a cloned cDNA sequence. The predicted leader PGPSLHF of the human BAD92446 sequence preceding the tryptic cleavage site (Arg) is not found in the chimpanzee sequence, which has an in-frame Met codon 210 amino acids upstream. The chimpanzee Thy1 C-terminus is the Leu-rich version of the human AAA61180 sequence. Thus, more research is needed to characterize this human variant CD90 protein. The existence of a CD90 variant would enhance the value of CD90 as a biomarker because it can be distinguished from the CD90 synthesized by white blood cells. In the PeptideAtlas database (http:// www.peptideatlas.org/), this variant CD90 peptide was not scored in large-scale mass spectrometry analysis of healthy human plasma. A follow-up study is being designed to examine the clinical utility of a CD90 urine test. Monoclonal antibodies need to be developed that can recognize specifically this CD90 variant.

In summary, we have identified a stromal cell type $\left(\mathrm{CD}^{+}\right.$fibroblasts) and a molecule (CD90) that distinguishes cancer-associated stroma from 'benign' stroma in the prostate. CD90 can be used to isolate cancer-associated stromal cells by flow or magnetic bead sorting, or immunofluorescencedirected laser microdissection to begin to understand mechanisms by which the stroma affects growth and progression of prostate cancer. In addition, CD90 might serve as a molecular target for therapy to regulate the growth of primary prostate cancer and as a diagnostic tool for atypical foci of prostate glands that are challenging to diagnose definitively using currently available histologic tools. We are beginning to collect these specimens in the frozen format because we are limited by the absence of an anti-CD90 antibody that works in sections of formalin-fixed, paraffin-embedded tissue. As all our studies were conducted on frozen sections of clearly diagnosable prostate cancer, the histology of most of the samples is of insufficient quality to identify 'atypical glands.' 


\author{
CD90/THY1 \\ AAA61180: MNLAISIALLLTVLQVSR----PPISSQNVTVLRDKLVKCEGISLLAQNTSWLLLLLLSLSLLQATDFMSL \\ BAD92446: PGPSLHFRWVGGPCAAGPSSLKEALLVPVLQVSR-----PPISSQNVTVLRGETSP
}

\begin{tabular}{|c|c|c|c|c|c|c|}
\hline+ & A & & & & & \\
\hline 61 & & $\mathrm{CA}$ & $\overline{G G C}$ & GCC & & \\
\hline 21 & $\mathrm{TT}$ & & & $\mathrm{TTG}$ & & \\
\hline 81 & & $\mathrm{TC}$ & $\mathrm{CTG}$ & GAC & & \\
\hline 41 & & & & & & \\
\hline 301 & & AACTCCCT & AGATCTCCT & & & $\lambda$ \\
\hline 361 & & & GGGT & & & \\
\hline 21 & & & & & & \\
\hline 81 & & & & & & \\
\hline 541 & & & & & & \\
\hline 01 & & & & & & \\
\hline 61 & $\mathrm{CT}$ & & & $\mathrm{CTT}$ & & \\
\hline 721 & & & & & & \\
\hline 781 & $T$ & & A & & & \\
\hline 41 & $\mathrm{CC}$ & ТСТССТСС & GTCA & & & \\
\hline 01 & & & C & & & \\
\hline 51 & & & & & & \\
\hline & & & GGT & & & \\
\hline & & & & & & \\
\hline & A & & & & & \\
\hline & TC & GTG & A & & & \\
\hline & C & & $\mathrm{GC}$ & & & \\
\hline & & GGG & & & & \\
\hline & & & & & & \\
\hline & & & & & & \\
\hline & & & & & & \\
\hline & & एT & A & & & \\
\hline & A & CC & & & & \\
\hline & & & & & & \\
\hline & & & & & & \\
\hline & & & & & & \\
\hline & & & & & & \\
\hline & A & & GG & & & \\
\hline & & & & & & \\
\hline & & & & $\mathrm{CT}$ & & \\
\hline & & & & & & \\
\hline & & & & & & \\
\hline & & & & & & \\
\hline & & & & & & \\
\hline & & & & & & \\
\hline & & & & & & \\
\hline & & & & & STGCCT & \\
\hline 521 & $\mathrm{ECC}$ & $A G$ & ССТGCCTTC & АСтТ & TGCGGGGCC & $\mathrm{AAC}$ \\
\hline & 110 & & TT & & GCTTTTCCC & \\
\hline & & & & & & \\
\hline & & & & & & \\
\hline & & & & & & \\
\hline
\end{tabular}

Figure 7 CD90 gene structure. The top panel shows the amino-acid sequence difference (in red) between the T-cell CD90 (AAA61180) and CD90 variant (BAD92446). In the AAA61180 sequence, MNLAISIALLLTVLQVSR constitutes the signal sequence. The bottom panel shows the gene arrangements of these two CD90 coding regions: nucleotides in red are for the T-cell CD90, and nucleotides in green are for variant CD90; common coding sequence is in blue. The start codon of AAA61180 and the stop codons of the two coding regions are underlined. A stop codon (TAG) precedes the CD90 variant coding sequence. Sequences of the CD90 primers in RT-PCR analysis are highlighted in gray. 


\section{Acknowledgements}

We thank Dr Laura Pascal for comments. This work was supported by grants CA111244 (EDRN), CA85859, and P50 CA097186 (Pacific Northwest Prostate Cancer SPORE) from NIH, the Prostate Cancer Foundation, and NIH NCRR center grant RR018522. Part of the experimental work was performed in the Environmental Molecular Sciences Laboratory, a US Department of Energy (DOE) Office of Biological and Environmental Research national scientific user facility on the Pacific Northwest National Laboratory (PNNL) campus. PNNL is a multi-program national laboratory operated by Battelle for the DOE under Contract DE-AC05-76RLO 1830.

\section{Disclosure/conflict of interest}

The authors declare no conflict of interest.

\section{References}

1 Cunha GR, Ricke W, Thomson A, et al. Hormonal, cellular, and molecular regulation of normal and neoplastic prostatic development. J Steroid Biochem Mol Biol 2004;92:221-236.

2 Sung SY, Chung LW. Prostate tumor-stroma interaction: molecular mechanisms and opportunities for therapeutic targeting. Differentiation 2002;70: 506-521.

3 Liu AY, True LD. Characterization of prostate cell types by CD cell surface molecules. Am J Pathol 2002;160: 37-43.

4 Liu AY, Roudier MP, True LD. Heterogeneity in primary and metastatic prostate cancer as defined by cell surface CD profile. Am J Pathol 2004;165: 1543-1556.

5 Zhang H, Li XJ, Martin DB, et al. Identification and quantification of N-linked glycoproteins using hydrazide chemistry, stable isotope labeling and mass spectrometry. Nat Biotechnol 2003;21:660-666.

6 Liu AY, Zhang H, Sorensen CM, et al. Analysis of prostate cancer by proteomics using tissue specimens. J Urol 2005;173:73-78.

7 Tian Y, Zhou Y, Elliott S, et al. Solid-phase extraction of N-linked glycopeptides. Nat Protocols 2007;2: 334-339.

8 Zhang H, Liu AY, Loriaux P, et al. Mass spectrometric detection of tissue proteins in plasma. Mol Cell Proteomics 2007;6:64-67.

9 Eng J, McCormack AL, Yates JR. An approach to correlate tandem mass spectral data of peptides with amino acid sequences in a protein database. J Am Mass Spectrom 1994;5:976-989.

10 Keller A, Nesvizhskii AI, Kolker E, et al. Empirical statistical model to estimate the accuracy of peptide identification made by MS/MS and database search. Anal Chem 2003;74:5383-5392.

11 Bause E. Structural requirements of $N$-glycosylation of proteins. Studies with proline peptides as conformational probes. Biochem J 1983;209:331-336.
12 Liu AY, True LD, LaTray L, et al. Cell-cell interaction in prostate gene regulation and cytodifferentiation. Proc Natl Acad Sci 1997;94:10705-10710.

13 Emmert-Buck MR, Bonner RF, Smith PD, et al. Laser capture microdissection. Science 1996;274: 998-1001.

14 von Haller PD, Yi E, Donohoe S, et al. The application of new software tools to quantitative protein profiling via isotope-coded affinity tag (ICAT) and tandem mass spectrometry: I. Statistically annotated datasets for peptide sequences and proteins identified via the application of ICAT and tandem mass spectrometry to proteins copurifying with $\mathrm{T}$ cell lipid rafts. Mol Cell Proteomics 2003;2:426-427.

15 von Haller PD, Yi E, Donohoe S, et al. The application of new software tools to quantitative protein profiling via isotope-coded affinity tag (ICAT) and tandem mass spectrometry: II. Evaluation of tandem mass spectrometry methodologies for large-scale protein analysis, and the application of statistical tools for data analysis and interpretation. Mol Cell Proteomics 2003;2: 428-442.

16 von Haller PD, Donohoe S, Goodlett DR, et al. Mass spectrometric characterization of proteins extracted from Jurkat $\mathrm{T}$ cell detergent-resistant membrane domains. Proteomics 2001;1:1010-1021.

17 Pascal LE, Goo Y, Vêncio RZN, et al. Gene expression down-regulation in $\mathrm{CD}^{+} 0^{+}$prostate tumor-associated stromal cells involves potential organ-specific genes. BMC Cancer 2009;9:317.

18 Pascal LE, Vêncio RZN, Page LS, et al. Gene expression relationship between prostate cancer cells of Gleason 3, 4 and normal epithelial cells as revealed by cell type-specific transcriptomes. BMC Cancer 2009;9:452.

19 Koumas L, Smith TJ, Phipps RP. Fibroblast subsets in the human orbit: Thy-1 $1^{+}$and Thy-1- $1^{-}$subpopulations exhibit distinct phenotypes. Eur J Immunol 2002;32: 477-485.

20 Koumas L, Smith TJ, Feldon S, et al. Thy-1 expression in human fibroblast subsets defines myofibroblastic or lipofibroblastic phenotypes. Am J Pathol 2003;163: 1291-1300.

21 Zhou Y, Hagood JS, Murphy-Ullrich JE. Thy-1 expression regulates the ability of rat lung fibroblasts to activate transforming growth factor- $\beta$ in response to fibrogenic stimuli. Am J Pathol 2004;165: 659-669.

22 Fries KM, Sempowski GD, Gaspari AA, et al. CD40 expression by human fibroblasts. Clin Immunol Immunopathol 1995;77:42-51.

23 King AE, Kelly RW, Critchley HO, et al. CD40 expression in uterine tissues: a key regulator of cytokine expression by fibroblasts. J Clin Endocrinol Metab 2001;86:405-412.

24 Hayward SW, Grossfeld GD, Tlsty TD, et al. Genetic and epigenetic influences in prostatic carcinogenesis. Int J Oncol 1998;13:35-47.

25 Zhang Y, Nojima S, Nakayama H, et al. Characteristics of normal stromal components and their correlation with cancer occurrence in human prostate. Oncol Rep 2003;10:207-211.

26 Olapade-Olaopa EO, MacKay EH, Taub NA, et al. Malignant transformation of human prostatic epithelium is associated with the loss of androgen receptor immunoreactivity in the surrounding stroma. Clin Cancer Res 1999;5:569-576. 
27 Tuxhorn JA, Ayala GE, Smith MJ, et al. Reactive stroma in human prostate cancer: induction of myofibroblast phenotype and extracellular matrix remodeling. Clin Cancer Res 2002;8:2912-2923.

28 Oudes AJ, Campbell DS, Sorensen CM, et al. Transcriptomes of human prostate cells. BMC Genomics 2006;7:92.

29 Liu AY, LaTray L, van den Engh G. Changes in cell surface molecules associated with in vitro culture of prostatic stromal cells. Prostate 2000;45: 303-312.

30 Saalbach A, Wetzig T, Haustein UF, et al. Detection of human soluble Thy-1 in serum by ELISA. Cell Tissue Res 1999;298:307-315.

31 Seki T, Spurr N, Obata F, et al. The human Thy-1 gene: structure and chromosomal location. Proc Natl Acad Sci 1985;82:6657-6661. 